\title{
Subcortical Aphasia After Stroke
}

\author{
Eun Kyoung Kang, MD, PhD ${ }^{1}$, Hae Min Sohn, SLP ${ }^{2}$, Moon-Ku Han, MD, PhD ${ }^{3}$, Nam-Jong Paik, MD, $\mathrm{PhD}^{4}$ \\ ${ }^{1}$ Department of Rehabilitation Medicine, Kangwon National University School of Medicine, Kangwon National University \\ Hospital, Chuncheon; ${ }^{2}$ Department of Rehabilitation Medicine, Seoul National University Bundang Hospital, Seongnam; \\ ${ }^{3}$ Department of Neurology, Seoul National University Bundang Hospital, Seoul National University College of Medicine, \\ Seongnam; ${ }^{4}$ Department of Rehabilitation Medicine, Seoul National University Bundang Hospital,
} Seoul National University College of Medicine, Seongnam, Korea

\begin{abstract}
Objective To evaluate the types and severity of subcortical aphasia after stroke and to determine the predictors of the degree of aphasic impairment.

Methods Medical records of 38 patients with post-stroke subcortical aphasia (19 males; mean age, $61.7 \pm 13.8$ years) were reviewed retrospectively with respect to the following tests: the Korean version of the Western Aphasia Battery (K-WAB), the Korean version of the Modified Barthel Index (K-MBI), and the Fugl-Meyer Index (FMI). The severity of aphasia was evaluated by the aphasia quotient (AQ) and the language quotient (LQ).

Results Anomic aphasia was the most frequent type of aphasia $(n=15,39.5 \%)$, and the lesion most frequently observed in subcortical aphasia was located in the basal ganglia $(n=19,50.0 \%)$. Patients with lesions in the basal ganglia exhibited the lowest scores on the FMI for the upper extremities $(p=0.04)$. Severity of aphasia was significantly correlated with the K-MBI (Pearson correlation coefficient: $\gamma=0.45, p=0.01$ for AQ and $\gamma=0.53$, $\mathrm{p}=0.01$ for LQ) and FMI scores for the lower extremities $(\gamma=0.43, \mathrm{p}=0.03$ for AQ and $\gamma=0.49, \mathrm{p}=0.05$ for LQ). In a multivariate logistic regression analysis, K-MBI remained the only explanatory variable closely associated with aphasia severity.

Conclusion This study showed the general characteristics of post-stroke subcortical aphasia, and it revealed that K-MBI was an associated and explanatory factor for aphasia severity.
\end{abstract}

Keywords Aphasia, Stroke, Basal ganglia, Cerebrovascular disease, Language tests, Association

\section{INTRODUCTION}

Aphasia is an impairment in receptive and expressive language modalities caused by damage to brain areas that are primarily responsible for language function.
Although aphasia is traditionally defined as cortical dysfunction, many studies have reported the occurrence of aphasia in isolated subcortical brain lesions [1-6]. Subcortical aphasia is a condition characterized by partial or total loss of the ability to communicate verbally and it

Received November 14, 2016; Accepted February 13, 2017

Corresponding author: Nam-Jong Paik

Department of Rehabilitation Medicine, Seoul National University Bundang Hospital, Seoul National University College of Medicine, 82 Gumi-ro 173beon-gil, Bundang-gu, Seongnam 13620, Korea. Tel: +82-31-787-7731, Fax: +82-31-787-4056, E-mail: njpaik@snu.ac.kr

ORCID: Eun Kyoung Kang (http://orcid.org/0000-0001-5315-1361); Hae Min Sohn (http://orcid.org/0000-0003-3545-3248); Moon-Ku Han (http:// orcid.org/0000-0003-0166-387X); Nam-Jong Paik (http://orcid.org/0000-0002-5193-8678).

(c) This is an open-access article distributed under the terms of the Creative Commons Attribution Non-Commercial License (http://creativecommons.org/ licenses/by-nc/4.0) which permits unrestricted noncommercial use, distribution, and reproduction in any medium, provided the original work is properly cited. Copyright (C) 2017 by Korean Academy of Rehabilitation Medicine 
develops as a result of damage to subcortical brain areas without loss of cortical function in Broca's or Wernicke's areas. Subcortical aphasia can result from lesions in the basal ganglia, white matter tracts, or thalamus [7-10].

Various language abnormalities develop after stroke as a function of the affected subcortical brain region; striato-capsular aphasia is associated with impaired executive language functions such as word fluency and sentence generation, but it largely spares responsive language functions such as comprehension, repetition, and naming [11]. Thalamic aphasia may produce dysfunction at the prelinguistic level, such as impairments in concept generation [12] and dysfunction in the control of preformed speech patterns [13]. For aphasia related to white matter lesions, the primary language dysfunction is an impairment in speech motor output [14].

Previous studies have reported that the type and severity of aphasia may vary following subcortical stroke $[12,15]$, but the pattern of symptoms and the factors associated with the severity of aphasia have not been fully explored. In the present study, we aimed to evaluate the types and severity of post-stroke subcortical aphasia by using standardized language evaluation tools and to identify the factors associated with the severity of subcortical aphasia.

\section{MATERIALS AND METHODS}

Medical records of patients with a first-time stroke, admitted to Seoul National University Bundang Hospital from June 2003 to June 2010, were reviewed retrospectively. After reviewing the results of the Korean version of the Western Aphasia Battery (K-WAB) [16], post-stroke aphasia patients with subcortical brain lesions were included. Patients were excluded from the study if they had accompanying (1) cortical aphasia, (2) traumatic brain injury, (3) a brain tumor, (4) a neurodegenerative disease, or (5) if they were found to have suffered multiple strokes. This study was approved by the Institutional Review Board of Seoul National University Bundang Hospital (IRB No. B-1101-120-110). Lesion locations were identified after a review of patients' brain MRIs.

Aphasia was assessed using K-WAB [16] administered by a speech-language pathologist (SLP) (number of poststroke days prior to evaluation, $29.9 \pm 23.0$ days). The KWAB consists of evaluation of specific components of oral language (subtests) such as spontaneous speech, auditory verbal comprehension, repetition, naming, reading and writing. The scores on these specific components were used for the classification of the aphasia subtype, according to Kertesz's method [17]. For evaluation of the severity of aphasia, which was based on oral language profiles of K-WAB, the aphasia quotient (AQ), and the language quotient (LQ) were used [18]. The AQ was calculated using the following formula:

$\mathrm{AQ}=($ fluency score + comprehension/20+repetition/10+ naming/10) $\times 2$

The LQ reflects reading and writing performance as well as auditory comprehension and oral expression. The LQ was calculated using the following formula [19]:

LQ=fluency score+comprehension/10+repetition/10+ naming/10+reading/5+writing/5

Additionally, medical records for the functional status were also reviewed: the Korean version of the Modified Barthel Index (K-MBI) [20], which was used to measure performance in activities of daily living; and the FuglMeyer Index of sensorimotor function (FMI) for the upper (UEx) and lower extremities (LEx) [21]. Handedness was determined by assessing the hand used for writing, feeding, throwing, and cutting with knives and scissors. Left- versus right-handedness was determined if a subject exhibited a preference for the same hand in more than 3 of 5 questions taken from the Edinburgh Handedness Inventory [22]. Patients were stratified by gender, handedness (right, left, or both), level of education, and type of stroke (infarction or hemorrhage).

To verify the effect of sex and stroke etiology on language profiles, an unpaired Student t-test was used. For comparisons between the clinical characteristics (age, post-stroke evaluation days, duration of education) and K-WAB scores for independent groups of aphasia type and brain lesion, an analysis of variance (ANOVA) was performed. Post-hoc analysis was performed by using Fisher LSD method to assess inter-group differences. Pearson correlation coefficient was used to evaluate the correlation between aphasia severity (AQ and LQ) and clinical characteristics. A multivariate logistic regression analysis (forward, stepwise) was performed to determine 
the factors associated with aphasia severity. All statistical tests were two-tailed, and p-values $<0.05$ were considered statistically significant. Values were presented as mean \pm standard deviation. Statistical analyses were carried out using SPSS for Windows ver. 20.0 (IBM, Armonk, NY, USA).

\section{RESULTS}

Thirty-eight aphasic patients (19 males) with left subcortical stroke were enrolled in the study. Seventeen (44.7\%) patients had infarcts and 21 (55.3\%) patients had hemorrhages. The mean age was $61.7 \pm 13.8$ years. All patients were right-handed. The mean post-stroke duration was 29.9 \pm 23.0 days (range, 6-99 days). The mean years of education were $11.1 \pm 5.0$ years (range 0 - 18 years).

The types of aphasia, in order of the most to the least common, were anomic aphasia $(n=15,39.5 \%)$, global aphasia $(n=6,15.8 \%)$, Broca's aphasia $(n=6,15.8 \%)$, Wernicke's aphasia $(n=6,15.8 \%)$, mixed transcortical aphasia $(\mathrm{n}=3,7.9 \%)$, and transcortical motor aphasia $(\mathrm{n}=2,5.3 \%)$. Among the types of aphasia, there were no significant differences in age, post-stroke evaluation days, MBI, FMIUEx, FMI-LEx, or length of education. There were very few cases with mixed transcortical aphasia $(n=3)$, transcortical motor aphasia $(n=2)$ and basal ganglia plus thalamic lesion aphasia $(n=1)$ to perform post-hoc analyses.

The K-WAB revealed that patients with anomic aphasia showed significantly higher scores for fluency, comprehension, repetition, naming, reading, writing, $\mathrm{AQ}$, and LQ than those with any other type of aphasia $(\mathrm{p}<0.05$ by LSD analysis). Patients with global aphasia presented the lowest scores for comprehension, repetition, naming, reading, AQ, and LQ ( $<<0.05$ by LSD analysis). Patients with Broca's aphasia had significantly higher comprehension scores than those with Wernicke's aphasia ( $p=0.02$ by LSD analysis), while they had significantly lower scores for fluency, repetition, and AQ ( $<<0.05$ by LSD analysis) (Table 1).

Patients with stroke confined to the basal ganglia scored significantly lower on the FMI-UEx than patients with stroke confined to the white matter ( $p=0.03$ by LSD analysis) or white matter plus basal ganglia $(\mathrm{p}=0.04$ by LSD analysis) lesions. However, there were no significant differences in age, sex, post-stroke delay in onset, years of education, or language profiles between brain lesions
( $p>0.05)$. Patients with aphasia associated with lesions in the basal ganglia showed worse AQ $(38.8 \% \pm 25.7 \%$ vs. $61.3 \% \pm 32.4 \%$ vs. $61.7 \% \pm 32.1 \%)$ and LQ $(40.4 \% \pm 27.9 \%$ vs. $62.2 \% \pm 35.8 \%$ vs. $63.9 \% \pm 35.2 \%$ ) compared to those with aphasia associated with white matter and thalamic lesions, respectively, but this difference was not statistically significant (Table 2). There were no statistically significant differences in language profiles according to sex and stroke etiology (infarction vs. hemorrhage) $(\mathrm{p}>0.05)$ (data not shown).

Patients with lesions in the basal ganglia $(n=19)$ were found to have either anomic aphasia $(n=5)$, global aphasia $(n=4)$, Broca's aphasia $(n=6)$, or Wernicke's aphasia $(\mathrm{n}=4)$. Patients with white matter lesions $(\mathrm{n}=8)$ had either anomic aphasia $(n=5)$, global aphasia $(n=1)$, Wernicke's aphasia $(n=1)$, or mixed transcortical aphasia $(n=1)$. Basal ganglia and white matter lesions $(n=3)$ were associated with anomic aphasia $(n=1)$, mixed transcortical aphasia $(n=1)$, and transcortical motor aphasia $(n=1)$. Patients with thalamic lesions $(n=7)$ had either anomic aphasia $(n=4)$, global aphasia $(n=1)$, Wernicke's aphasia $(n=1)$, or mixed transcortical aphasia $(n=1)$. When a thalamic lesion was accompanied by a lesion in the basal ganglia, transcortical motor aphasia was observed. We also compared the patients in the two lesion groups: basal ganglia $(n=23)$ vs. non-basal ganglia $(n=15)$; white matter $(n=11)$ vs. non-white matter $(\mathrm{n}=27)$; and thalamic $(\mathrm{n}=8)$ vs. nonthalamic $(n=30)$ (Table 3$)$. These comparisons did not yield any significant differences in language profiles or clinical characteristics, except for the delay in onset of aphasia after stroke $(43.9 \pm 5.8$ days vs. $26.1 \pm 17.1$ days, respectively, $\mathrm{p}=0.05)$ only in the thalamic $(\mathrm{n}=8)$ and nonthalamic $(n=30)$ comparison.

Pearson correlation coefficients between language profiles and demographic data are presented in Table 4. There were significant relationships between MBI, FMILEx, and aphasia severity (AQ and LQ) (Fig. 1). After multivariate logistic regression (forward, stepwise), KMBI remained the only independent predictive factor for aphasia severity ( $\mathrm{p}=0.01)$ (Table 5).

\section{DISCUSSION}

In our study, anomic aphasia was the most frequent subtype of subcortical aphasia, while global aphasia was the most common type of cortical aphasia. The severity 


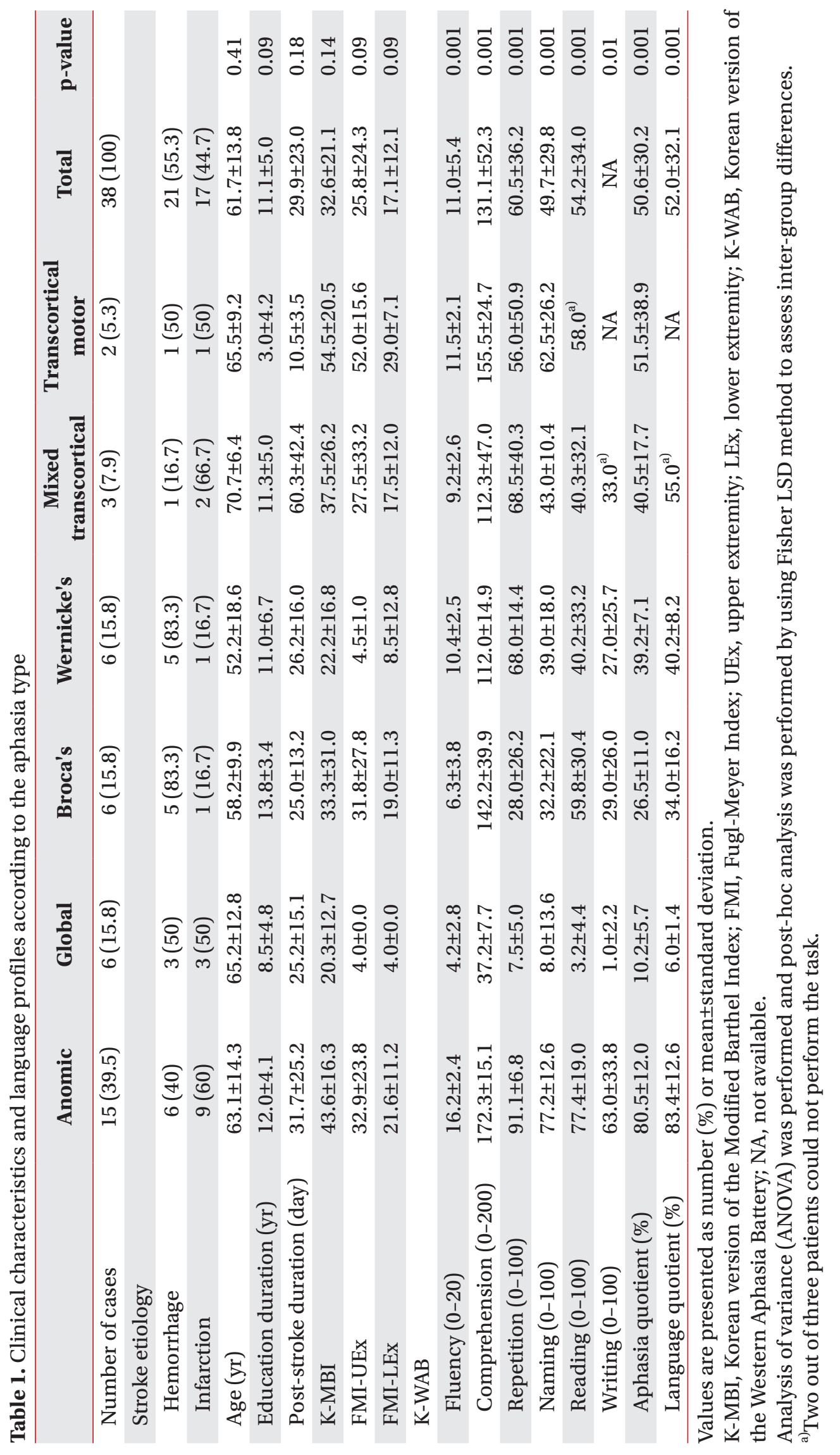




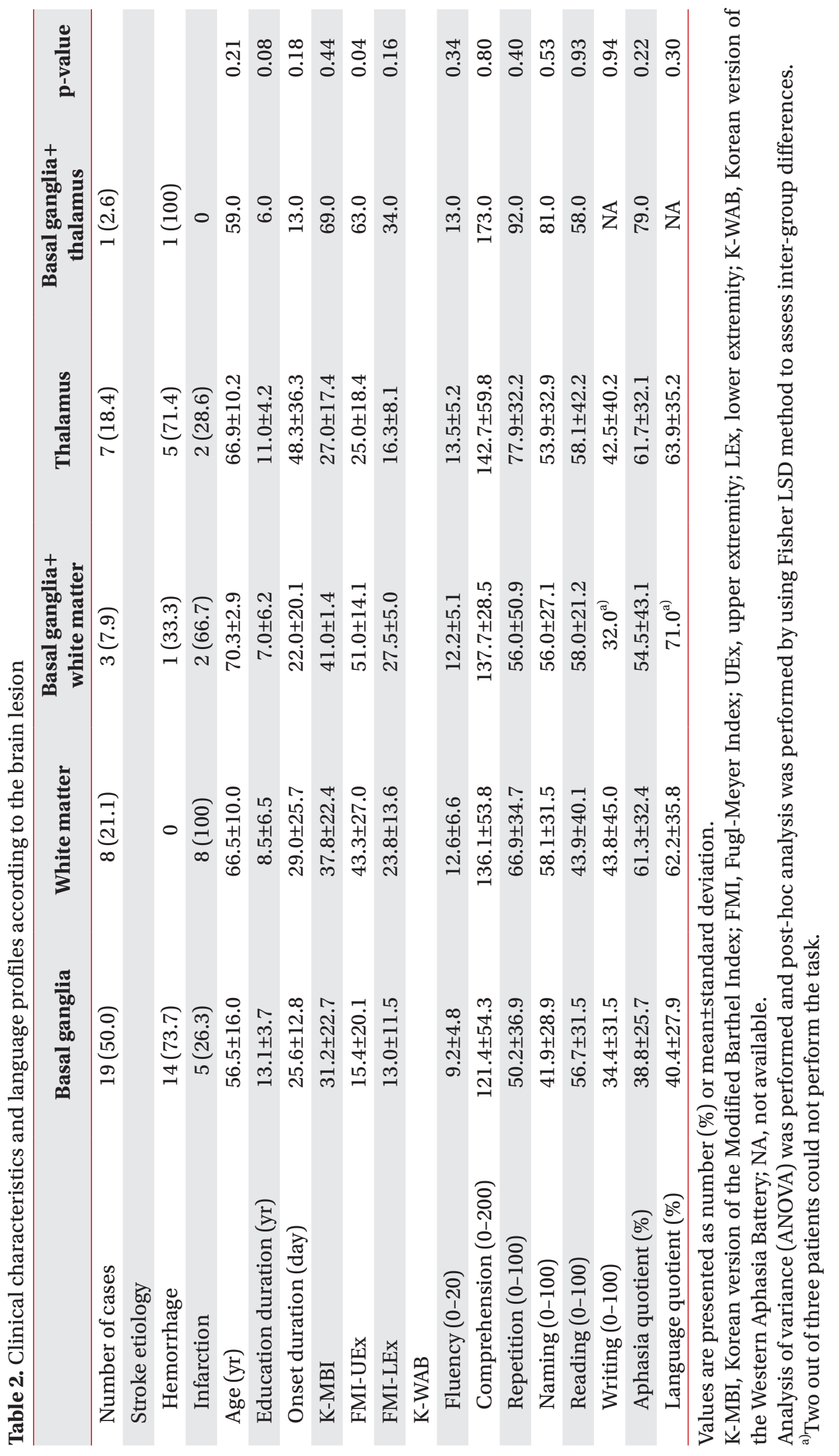


Table 3. Aphasia type according to the brain lesions

\begin{tabular}{|lccccccc}
\hline \multicolumn{1}{c}{ Brain lesion } & $\begin{array}{c}\text { Anomic } \\
(\mathbf{n}=\mathbf{1 5})\end{array}$ & $\begin{array}{c}\text { Global } \\
(\mathbf{n}=\mathbf{6})\end{array}$ & $\begin{array}{c}\text { Broca's } \\
(\mathbf{n}=\mathbf{6})\end{array}$ & $\begin{array}{c}\text { Wernicke's } \\
(\mathbf{n}=\mathbf{6})\end{array}$ & $\begin{array}{c}\text { Mixed } \\
\text { transcortical } \\
(\mathbf{n}=\mathbf{3})\end{array}$ & $\begin{array}{c}\text { Transcortical } \\
\text { motor } \\
(\mathbf{n}=\mathbf{2})\end{array}$ & Total \\
\hline Basal ganglia & 5 & 4 & 6 & 4 & 0 & 0 & 19 \\
\hline White matter & 5 & 1 & 0 & 1 & 1 & 0 & 8 \\
\hline Basal ganglia+white matter $\left.{ }^{\mathrm{a}}\right)$ & 1 & 0 & 0 & 0 & 1 & 1 & 3 \\
\hline Thalamus & 4 & 1 & 0 & 1 & 1 & 0 & 7 \\
\hline Basal ganglia+thalamus, ${ }^{\mathrm{a}, \mathrm{b})}$ & 0 & 0 & 0 & 0 & 0 & 1 & 1 \\
\hline Binary classification & & & & & & & \\
\hline Basal ganglia & 6 & 4 & 6 & 4 & 1 & 2 & 23 \\
\hline Non-basal ganglia & 9 & 2 & 0 & 2 & 2 & 0 & 15 \\
\hline White matter & 6 & 1 & 0 & 1 & 2 & 1 & 11 \\
\hline Non-white matter & 9 & 5 & 6 & 5 & 1 & 1 & 27 \\
\hline Thalamic & 4 & 1 & 0 & 1 & 1 & 1 & 8 \\
\hline Non-thalamic & 11 & 5 & 6 & 5 & 2 & 1 & 30 \\
\hline
\end{tabular}

a) These cases were incorporated into the basal ganglia or white matter lesion group.

${ }^{b)}$ This case was incorporated into the thalamic lesion group.

Table 4. Pearson correlation coefficients ( $\mathrm{p}$-value) between aphasia severity and demographic data

\begin{tabular}{lcccccc}
\hline & Age & Onset duration & K-MBI & FMI-UEx & FMI-LEx & $\begin{array}{c}\text { Education } \\
\text { duration }\end{array}$ \\
\hline Aphasia quotient $(\%)$ & $0.01(0.93)$ & $0.08(0.63)$ & $0.45(0.01)$ & $0.32(0.12)$ & $0.43(0.03)$ & $0.17(0.32)$ \\
\hline Language quotient $(\%)$ & $-0.05(0.79)$ & $0.11(0.59)$ & $0.53(0.01)$ & $0.34(0.18)$ & $0.49(0.05)$ & $0.25(0.21)$ \\
\hline
\end{tabular}

K-MBI, Korean version of the Modified Barthel Index; FMI, Fugl-Meyer Index; UEx, upper extremity; LEx, lower extremity.

of subcortical aphasia was milder than that of cortical aphasia as reported in our previous study [23].

The aim of this study was to evaluate the linguistic features of post-stroke subcortical aphasia, and to determine the predictors (i.e., lesion type and clinical characteristics) of the degree of aphasic impairment. We performed standardized language evaluation tests in post-stroke subcortical aphasia patients.

Cortical aphasia has been reported to correlate well with the type and severity of aphasia [23]. However, our data indicate that subcortical aphasias do not show any correlation between lesion location and the type and severity of aphasia. These findings are consistent with previous studies [1]. The fact that the same subcortical brain lesion elicited different types of aphasia suggests that rather than direct action, essential components of language networks, subcortical structures have an indirect and multifaceted effect on language $[1,8]$.

In previous studies, the most common type of cortical aphasia reported were global aphasia [23] and anomic aphasia $[11,24]$. Subcortical aphasia is usually associated with a higher language performance compared to cortical aphasia $[23,25]$. The present study confirmed that anomic aphasia is the most frequent type of subcortical aphasia, and that this type of aphasia exhibits a higher level of language profile compared to cortical aphasia as noted in our previous study [23]. Additionally, patients with global aphasia (AQ, $10.2 \% \pm 5.7 \%$ ) presented the worst language features, while those with anomic aphasia (AQ, $80.5 \% \pm 12.0 \%$ ) presented the mildest impairment.

In this study, subcortical aphasia was classified into striato-capsular aphasia, thalamic aphasia, and aphasia associated with white matter lesions. Generally, cortical aphasia is caused by a brain lesion in Broca's or Wernicke's area, which is supplied by the middle cerebral artery (MCA). The incidence of aphasia as a result of a defect in the posterior cerebral arterial (PCA) supply is considerably lower [26]. Consistent with this finding, in 

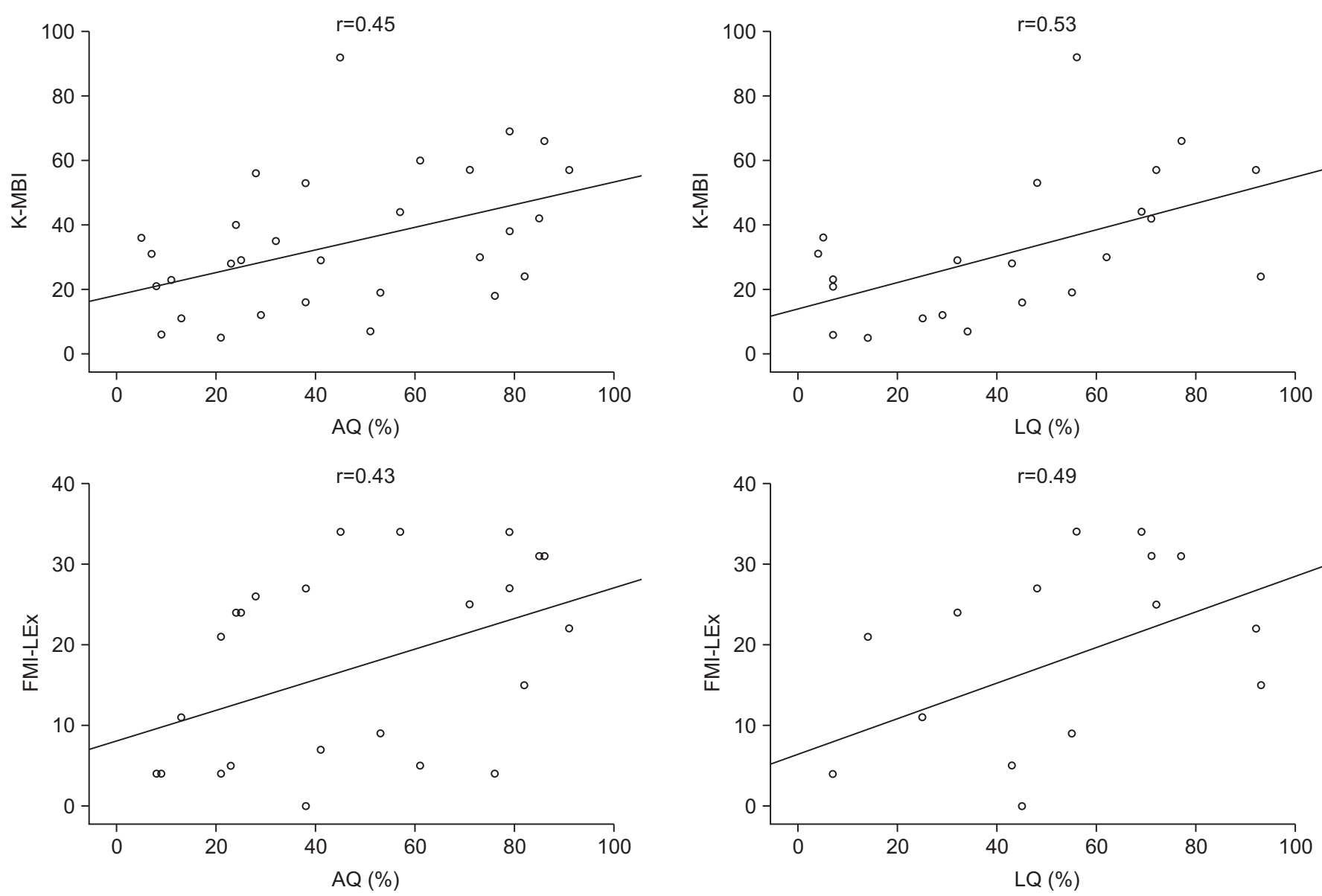

Fig. 1. Significant Pearson correlations of the aphasia quotient (AQ) and language quotient (LQ) with Korean version of the Modified Barthel Index (K-MBI) and Fugl-Meyer Index of sensorimotor function (FMI) for the lower extremities (LEx).

Table 5. Multivariate analyses of factors related to the severity of subcortical aphasia

\begin{tabular}{cccc}
\hline Dependent variable & Explanatory variable & Standardized coefficient $(\boldsymbol{\beta})$ & p-value \\
\hline Aphasia quotient & K-MBI & 0.50 & 0.01 \\
Language quotient & K-MBI & 0.59 & 0.01 \\
\hline
\end{tabular}

K-MBI, Korean version of the Modified Barthel Index.

this study, we observed that subcortical aphasia caused by PCA occlusion (8 cases) was less frequent than subcortical aphasia caused by compromised MCA supply (30 cases). However, these results did not reflect the general tendency of brain lesions in subcortical aphasia.

The principal brain regions associated with language are cortical areas such as Broca's and Wernicke's areas. Usually, patients with thalamic aphasia showed more severe forms of defective comprehension and a reduction of spontaneous verbal output in comparison to those with other types of aphasia [12]. However, the language profiles in the present study were not significantly different between these groups. Binary classification (thalamic lesion vs. non-thalamic lesion) revealed that the poststroke evaluation delay in thalamic aphasia was significantly longer than that in non-thalamic aphasia, indicating the possibility of speech recovery in thalamic aphasia patients.

Aphasia has been associated with severe physical disability [27]. Moreover, severe aphasia has been associated with more profound impairments and an increased lack of autonomy in activities of daily living (ADL) than that in 
patients without aphasia or with mild aphasia [28]. Consistent with these studies, aphasia has also been associated with more severe impairment in social activities and less complete recovery of social activities [29].

In the present study, MBI was associated with the severity of aphasia, and it was the factor that correlated with AQ and LQ. These results indicate a close relationship between aphasia and patients' functional status. Based on this finding, the severity of aphasia can affect the functional outcome after stroke [30] and aphasia might influence the extent of ADL dependency. Thus, speech therapy for subcortical aphasia patients could improve their functional status by improving their communication skills.

Patient age was not correlated with the severity of aphasia in the current study. Consistent with this finding, a previous study reported no age differences between patients with fluent or non-fluent aphasia after acute stroke [31]. We suggest that true aphasia may have been masked by temporary non-fluent aphasia in acute stage, which may be due to diaschisis or the penumbra phenomenon [31]. Additionally, sex was not a significant factor affecting the severity of aphasia [32]. The influence of stroke etiology had not been determined prior to the present study. Here, we report that the severity of aphasia does not appear to be influenced by stroke etiology.

Our study did not evaluate the relationship between the extent of subcortical lesions and aphasia severity, which is a significant limitation. Some previous studies have reported that subcortical lesion volume and aphasia severity are not correlated $[1,33]$, while other studies have reported that the severity of subcortical aphasia was closely related to the extent and severity of cerebral cortical hypoperfusion identified by brain perfusion SPECT $[4,12,34]$. Therefore, functional neuroimaging studies are needed to resolve this controversial issue. Additionally, this study used K-WAB as an aphasia evaluation tool, but we did not evaluate other speech dysfunctions such as hypophonia, dysarthria, or apraxia of speech, which may accompany subcortical stroke. Moreover, this study showed a wide range of post-stroke onset duration because of the retrospective chart review even though it was regarded as the subacute stroke phase. Thus, comprehensive language evaluation with prospective recruitment of patients should be considered in the future.

In summary, post-stroke subcortical aphasia caused variable language abnormalities, and it was not correlated with the lesion site or etiology. Subcortical aphasia was found to have different features of language profile from cortical aphasia, and aphasia severity was closely associated with K-MBI.

\section{CONFLICT OF INTEREST}

No potential conflict of interest relevant to this article was reported.

\section{REFERENCES}

1. Hillis AE, Barker PB, Wityk RJ, Aldrich EM, Restrepo L, Breese EL, et al. Variability in subcortical aphasia is due to variable sites of cortical hypoperfusion. Brain Lang 2004;89:524-30.

2. Alexander MP, Naeser MA, Palumbo CL. Correlations of subcortical CT lesion sites and aphasia profiles. Brain 1987;110(Pt 4):961-91.

3. Murdoch BE. Subcortical brain mechanisms in speech and language. Folia Phoniatr Logop 2001;53:233-51.

4. Choi JY, Lee KH, Na DL, Byun HS, Lee SJ, Kim H, et al. Subcortical aphasia after striatocapsular infarction: quantitative analysis of brain perfusion SPECT using statistical parametric mapping and a statistical probabilistic anatomic map. J Nucl Med 2007;48:194-200.

5. Jensen AM, Chenery HJ, Copland DA. A comparison of picture description abilities in individuals with vascular subcortical lesions and Huntington's disease. J Commun Disord 2006;39:62-77.

6. Gout A, Seibel N, Rouviere C, Husson B, Hermans B, Laporte $\mathrm{N}$, et al. Aphasia owing to subcortical brain infarcts in childhood. J Child Neurol 2005;20:1003-8.

7. Mohr JP, Watters WC, Duncan GW. Thalamic hemorrhage and aphasia. Brain Lang 1975;2:3-17.

8. Damasio AR, Damasio H, Rizzo M, Varney N, Gersh F. Aphasia with nonhemorrhagic lesions in the basal ganglia and internal capsule. Arch Neurol 1982;39:1524.

9. Naeser MA, Alexander MP, Helm-Estabrooks N, Levine HL, Laughlin SA, Geschwind N. Aphasia with predominantly subcortical lesion sites: description of three capsular/putaminal aphasia syndromes. Arch Neurol 1982;39:2-14.

10. Graff-Radford NR, Damasio H, Yamada T, Eslinger PJ, 
Damasio AR. Nonhaemorrhagic thalamic infarction. Clinical, neuropsychological and electrophysiological findings in four anatomical groups defined by computerized tomography. Brain 1985;108(Pt 2):485-516.

11. Mega MS, Alexander MP. Subcortical aphasia: the core profile of capsulostriatal infarction. Neurology 1994;44:1824-9.

12. Nadeau SE, Crosson B. Subcortical aphasia. Brain Lang 1997;58:355-402.

13. Schaltenbrand G. The effects on speech and language of stereotactical stimulation in thalamus and corpus callosum. Brain Lang 1975;2:70-7.

14. Staudt M, Grodd W, Niemann G, Wildgruber D, Erb M, Krageloh-Mann I. Early left periventricular brain lesions induce right hemispheric organization of speech. Neurology 2001;57:122-5.

15. Kennedy M, Murdoch BE. Chronic aphasia subsequent to striato-capsular and thalamic lesions in the left hemisphere. Brain Lang 1993;44:284-95.

16. Kim H, Na DL. Paradise Korean version of the Western Aphasia Battery. Seoul: Paradise Welfare Foundation; 2001.

17. Kertesz A. Aphasia and associated disorders: taxonomy, localization, and recovery. New York: Grune \& Stratton; 1979.

18. Kertesz A, Poole E. The aphasia quotient: the taxonomic approach to measurement of aphasic disability. Can J Neurol Sci 1974;1:7-16.

19. Shewan CM. The Language Quotient (LQ): a new measure for the Western Aphasia Battery. J Commun Disord 1986;19:427-39.

20. Jung HY, Park BK, Shin HS, Kang YK, Pyun SB, Paik NJ, et al. Development of the Korean version of modified barthel index (K-MBI): multi-center study for subjects with stroke. J Korean Acad Rehabil Med 2007;31:28397.

21. Sanford J, Moreland J, Swanson LR, Stratford PW, Gowland C. Reliability of the Fugl-Meyer assessment for testing motor performance in patients following stroke. Phys Ther 1993;73:447-54.

22. Williams SM. Factor analysis of the Edinburgh Hand- edness Inventory. Cortex 1986;22:325-6.

23. Kang EK, Sohn HM, Han MK, Kim W, Han TR, Paik NJ. Severity of post-stroke aphasia according to aphasia type and lesion location in Koreans. J Korean Med Sci 2010;25:123-7.

24. Kim DH, Kim MJ, Kwon HK, Lee HJ. Types, severity and prognostic factors in subcortical aphasia. J Korean Acad Rehabil Med 1999;23:478-84.

25. Devinsky O, D'Esposito M. Neurology of cognitive and behavioral disorders. New York: Oxford University Press; 2004.

26. Alexander MP. Aphasia: clinical and anatomic aspects. In: Feinberg TE, Farah MJ, editors. Behavioral neurology and neuropsychology. New York: McGrawHill; 1997. p. 133-49.

27. Wade DT, Hewer RL, David RM, Enderby PM. Aphasia after stroke: natural history and associated deficits. J Neurol Neurosurg Psychiatry 1986;49:11-6.

28. Kauhanen ML, Korpelainen JT, Hiltunen P, Maatta R, Mononen H, Brusin E, et al. Aphasia, depression, and non-verbal cognitive impairment in ischaemic stroke. Cerebrovasc Dis 2000;10:455-61.

29. Wade DT, Hewer RL, David RM, Enderby PM. Aphasia after stroke: natural history and associated deficits. J Neurol Neurosurg Psychiatry 1986;49:11-6.

30. Fang Y, Chen X, Li H, Lin J, Huang R, Zeng J. A study on additional early physiotherapy after stroke and factors affecting functional recovery. Clin Rehabil 2003; 17:608-17.

31. Pedersen PM, Vinter K, Olsen TS. Aphasia after stroke: type, severity and prognosis. The Copenhagen aphasia study. Cerebrovasc Dis 2004;17:35-43.

32. Godefroy O, Dubois C, Debachy B, Leclerc M, Kreisler A; Lille Stroke Program. Vascular aphasias: main characteristics of patients hospitalized in acute stroke units. Stroke 2002;33:702-5.

33. Kirk A, Kertesz A. Subcortical contributions to drawing. Brain Cogn 1993;21:57-70.

34. Weiller C, Willmes K, Reiche W, Thron A, Isensee C, Buell U, et al. The case of aphasia or neglect after striatocapsular infarction. Brain 1993;116(Pt 6):1509-25. 